\title{
Using Consistency-driven Pairwise Comparisons in Knowledge-based Systems
}

\author{
Waldemar W. Koczkodaj*and Michael W. Herman, Computer Science \\ Laurentian University, Sudbury, Ontario, Canada P3E 2C6 \\ http://www.laurentian.ca/math/wkoczkodaj.html \\ Marian Orlowski 'School of Information Systems \\ Queensland University of Technology, Brisbane, Q 4001, Australia \\ Orlowski@fit.qut.edu.au
}

August 13, 1997

\begin{abstract}
Knowledge acquisition often requires the assessment of qualitative stimuli (e.g., criteria or priorities) such as public safety or the degree of environmental damage. Weights, reflecting the relative importance of the objectives concerned are one of the most commonly used solutions for this kind of data. Subjective judgments involve inaccuracy (which is difficult to control) and consistency of judgments (which can be measured and used to influence the accuracy). The consistency-driven pairwise comparisons method is based on an inconsistency index and its use as a validation technique. Knowledge acquisition using the consistency-driven pairwise comparisons method, when supported by properly designed software, contributes to the improvement of the quality of knowledge-based systems.
\end{abstract}

Keywords: knowledge acquisition, knowledge validation, consistency-driven pairwise comparison, multicriteria evaluation, consistency, expert system.

\section{Knowledge Acquisition and Valida- tion}

Knowledge acquisition is the process of extracting, structuring and organizing knowledge from various sources of expertise. It may be defined as: the art of bringing the principles and tools of Artificial Intelligence research to bear on applications involving difficult problems that require knowledge of human experts for their solutions (see [3]). The technical issues of acquiring this knowledge, representing it, and using it appropriately to construct and explain the lines-of-reasoning are im-

*to the correspondence should be directed; partially supported by the Natural Sciences and Engineering Research Council of Canada (NSERC) and by the Ontario Heritage Corporation through the Ministry of Northern Development and Mines.

†partially supported by the Australian Research Council grant A49602509 portant problems in the design of knowledge- based systems.

Knowledge acquisition is performed throughout the systems development life cycle (SDLC). Also during the entire SDLC, the knowledge is validated and verified until its quality is acceptable for inclusion into a knowledge base. Knowledge is viewed as a collection of specialized facts, procedures, and judgment rules collected from many sources. These sources can be divided into two types: documented and undocumented. The latter resides in people's minds. Knowledge can be identified and collected by using any of the human senses. The multiplicity of sources and types of knowledge contributes to the complexity of knowledge acquisition.

Transferring information from one person to another is difficult for several reasons. Experts may lack time or be unwilling to cooperate. Testing and refining knowledge is complicated. Methods for knowledge elicitation might be poorly defined. System builders have a tendency to collect knowledge from a single source, although the relevant information may be scattered across several sources. Builders may attempt to collect documented knowledge rather than use experts. Consequently, the knowledge collected may be incomplete and/or inaccurate. It is difficult to recognize specific knowledge when it is mixed with irrelevant data.

The basic model of knowledge engineering is based on teamwork in which a knowledge engineer mediates between experts and the knowledge base. The knowledge engineer elicits knowledge from experts, refines it with them, and represents it in the knowledge base. The elicitation of knowledge from experts can be done manually or with the aid of computers.

The knowledge may be expressed, for example, by assessing preferences of stimuli (e.g., criteria, factors, or possible alternatives). When devising methods for formulating and assessing preferences, a knowledge engi- 
neer has to take into account the limitations in human capabilities for undertaking such an endeavour. One possible technique for extracting the expert's knowledge and preferences is based on the consistency-driven pairwise comparisons method.

\section{Basic Concepts of Pairwise Compar- isons}

The use of the consistency-driven pairwise comparisons method for knowledge acquisition and consistencydriven knowledge validation is proposed. The pairwise comparisons method (also known as paired comparisons) was introduced in embryonic form by Fechner (see [4]). After considerable extension it was formalized by Thurstone (see [14]). In 1977 Saaty ([12]) transformed the pairwise comparisons method to a useful tool by adding hierarchical structures (for larger $n$, the $O\left(n^{2}\right)$ complexity was a problem). The consistency-driven extension to the traditional pairwise comparisons method is based on the new definition of inconsistency (see [7] and most recently [9]).

This paper will demonstrate the consistency-driven pairwise comparisons method as a powerful knowledge acquisition technique in knowledge-based systems and data mining. The consistency-driven pairwise comparisons method processes statements expressed by experts about preferences and judgments. These statements are aligned to pairs of criteria or objectives. Assessments provided by experts are mapped into a numerical scale (see Table 1) for easier processing. Ordinal numbers are used to express relative preferences. It is necessary to stress that these numbers do not represent "an absolute" measure of the mapped stimuli since such a measure may not exist. For example, it is hard to define a standard measure for public safety but, it is still practical to relate it to environmental pollution for a given case or project.

Input data are arranged in a pairwise comparisons matrix $A=\left[a_{i j}\right]$, whose elements represent the intensities of an expert's preference between individual pairs of alternatives or criteria chosen from a given scale (in our case it is from 1 to 5 ).

A $n \times n$ pairwise comparisons matrix is thus defined as a square matrix $A=\left\{a_{i j}\right\}$ such that $a_{i j}>0$ for every $i, j=1, \ldots, n$. Each $a_{i j}$ expresses a relative preference of stimulus (or criterion) $s_{i}$ over stimulus $s_{j}$ for $i, j=$ $1, \ldots, n$ represented by numerical weights (positive real numbers) $w_{i}$ and $w_{j}$ respectively. The quotients $a_{i j}=$ $\frac{w_{i}}{w_{j}}$ form a pairwise comparisons matrix

$$
A=\left|\begin{array}{cccc}
1 & a_{12} & \cdots & a_{1 n} \\
\frac{1}{a_{12}} & 1 & \cdots & a_{2 n} \\
\vdots & \vdots & \vdots & \vdots \\
\frac{i}{a_{1 n}} & \frac{i}{a_{2 n}} & \cdots & 1
\end{array}\right|
$$

Table 1: Comparisons scale

\begin{tabular}{|c|c|c|}
\hline $\begin{array}{l}\text { Intensity } \\
\text { of } \\
\text { Impor- } \\
\text { tance }\end{array}$ & Definition & Explanation \\
\hline 1 & $\begin{array}{ll}\text { Equal } & \text { or } \\
\text { unknown } & \\
\text { importance } & \end{array}$ & $\begin{array}{l}\text { Two criteria con- } \\
\text { tributed equally to } \\
\text { the objective or it is } \\
\text { impossible to assess it }\end{array}$ \\
\hline 2 & $\begin{array}{l}\text { Moderate im- } \\
\text { portance of one } \\
\text { over another }\end{array}$ & $\begin{array}{l}\text { Experience and judg- } \\
\text { ments slightly favour } \\
\text { one stimulus over } \\
\text { another }\end{array}$ \\
\hline 3 & $\begin{array}{ll}\text { Essential } & \text { or } \\
\text { strong } & \\
\text { importance } & \end{array}$ & $\begin{array}{l}\text { Experience and judg- } \\
\text { ments strongly favour } \\
\text { one stimulus over } \\
\text { another }\end{array}$ \\
\hline 4 & $\begin{array}{l}\text { Demonstrated } \\
\text { or strong } \\
\text { importance }\end{array}$ & $\begin{array}{l}\text { One stimulus } \\
\text { is strongly favoured } \\
\text { and its dominance } \\
\text { is demonstrated in } \\
\text { practice }\end{array}$ \\
\hline 5 & $\begin{array}{l}\text { Absolute } \\
\text { importance }\end{array}$ & $\begin{array}{l}\text { The evidence favour- } \\
\text { ing one stimulus over } \\
\text { another is of the high- } \\
\text { est possible order of } \\
\text { affirmation }\end{array}$ \\
\hline $\begin{array}{l}1.5,3.1 \\
\ldots, \text {, etc. }\end{array}$ & $\begin{array}{l}\text { Intermediate } \\
\text { values between } \\
\text { the two adjacent } \\
\text { judgments } \\
\end{array}$ & $\begin{array}{l}\text { when compromise is } \\
\text { needed }\end{array}$ \\
\hline
\end{tabular}

A pairwise comparisons matrix $A$ is called reciprocal if $a_{i j}=\frac{i}{a_{j i}}$ for every $i, j=1, \ldots, n$ (then automatically $a_{i i}=1$ for every $i=1, \ldots, n$ which represents tho relative ratio of a stimulus with itself). A pairwise comparisons matrix $A$ is called consistent if $a_{i j} \cdot a_{j k}=a_{i k}$ holds for every $i, j, k=1, \ldots, n$ since $\frac{w_{i}}{2 w_{j}} \frac{w_{j}}{w_{k}}$ is expected to be equal to $\frac{w_{i}}{w_{k}}$. Although every consistent matrix is reciprocal, the converse is generally not true. In practice, comparing $s_{i}$ to $s_{j}, s_{j}$ to $s_{k}$, and $s_{i}$ to $s_{k}$ often results in inconsistency amongst the assessments in addition to their inaccuracy; however, the inconsistency may be computed and used to improve the accuracy since we can locate the most inconsistent judgements.

The first step in pairwise comparisons is to establish the relative preference of each combination of two criteria. A scale of an appropriate granularity can be used to compare all criteria in pairs. (We are using values from $[1,5]$; values from the interval $\left[\frac{1}{5}, 1\right]$ reflect inverse rela. tionships between criteria since $\left.\frac{w_{i}}{w_{j}}=\frac{1}{\frac{x_{j}}{w_{i}}}\right)$. It has been shown ([15]) that all reasonable scales are equivalent for a small enough inconsistency. The consistency-driven 
approach is based on the reasonable assumption that finding the most inconsistent judgements may lead to a reconsideration of judgements. This in turn contributes to the improvement of assessment accuracy. Consistency analysis is a dynamic process which is assisted by software.

Saaty's theorem ([12]) states that for every $n$ by $n$ consistent matrix $A=\left[a_{i j}\right]$ there exist positive real numbers $w_{1}, \ldots w_{n}$ (weights corresponding to criteria $\left.s_{1}, \ldots, s_{n}\right)$ such that $a_{i j}=w_{i} / w_{j}$ for every $i, j=$ $1, \ldots, n$. The weights $w_{i}$ are unique up to a multiplicative constant and are the components of the principal eigenvector corresponding to the largest eigenvalue of $A$. Saaty's eigenvector method is one of several techniques for computing the weights. They can also be computed by the least-squares-method (LSM) and the logarithmic-least-square-method (LLSM, also known as the method of row geometric means, GM). In fact, GM is the simplest and most effective method. A Monte Carlo experiment ([5]) demonstrated that the accuracy, that is, the distance from the original matrix $A$ to the matrix $A^{\prime}$ reconstructed from weights with elements $a_{i j}^{\prime}=\left[w_{i} / w_{j}\right]$, does not depend strongly on the method. There is, however, a strong relationship between the accuracy of the weights and the inconsistency of the pairwise comparisons matrix. The main focus of the consistency-driven approach is consistency analysis.

\section{Consistency Analysis}

Consistency analysis is critical to the approach presented here because the accuracy of the weights computed for not-so-inconsistent matrices depends strongly on the inconsistency ([5]). This makes the consistencydriven approach the next step in the development of the pairwise comparisons method. Assessing intangible stimuli (e.g., the degree of an environmental hazard or pollution factors) involves not only imprecise or inexact knowledge but also the inconsistency in our own judgments. The improvement of knowledge elicitation by controlling the inconsistency of experts' judgments is not only desirable but necessary.

Checking consistency in the consistency-driven pairwise comparisons method can be compared to checking that the divisor is not equal to 0 . It does not make mathematical sense to divide anything by 0 . The classical pairwise comparisons method is based on the assumption that the given reciprocal matrix is consistent (see [12]). Why can we simply assume that the reciprocal matrix is fully consistent? Requesting all the judgments to be consistent is not realistic since we wish to manage judgements which are subjective, inaccurate, and nearly always contain some kind of bias and imprecision. The inconsistency in subjective judgements is not unexpected. In fact it may even be perceived as a desirable indication that the data truly reflect reality (as opposed to data that have been 'doctored').
Given an $n \times n$ matrix $A$ that is not consistent, the pairwise comparisons method attempts to provide a consistent $n \times n$ matrix $A^{\prime}$ that differs from the matrix $A$ "as little as possible".

The definition of a global inconsistency of a pairwise comparisons matrix $A$ introduced by Saaty (see [12]) is based on eigenvalues and is given by the formula:

$$
c f=\frac{\lambda_{A}-\operatorname{order}(A)}{(\operatorname{order}(A)-1) \lambda_{\text {ratulom }}}
$$

where $\lambda$ is the largest eigenvalue of the reciprocal matrix A.

The above formula leads to some theoretical problems (see: [13] and [7]). First of all, $c f$ depends on the factor $\lambda_{\text {random }}$ (the mean value of the largest eigenvalues of a certain arbitrary number of randomly generated reciprocal matrices) which may vary. Factor $c f$ is also not a reliable discernibility index since it changes fast for small deviations of matrix elements in some cases). The biggest deficiency of the eigenvalue-based inconsistency index is its inability to locate the most inconsistent elements in the matrix since $c f$ is a global matrix characteristic (a just scaled eigenvalue). An improved definition of inconsistency (see [7]) is based on triads of the elements of the comparisons matrix $A$, which are associated by the consistency relation. A pairwise comparisons matrix of order 3 is reduced to the following basic reciprocal matrix:

$$
A 3=\left|\begin{array}{ccc}
1 & a & b \\
\frac{1}{a} & 1 & c \\
\frac{1}{b} & \frac{1}{c} & 1
\end{array}\right|
$$

where $a$ expresses an expert's relative preference of stimulus $s_{i}$, over $s_{j}, b$ expresses a preference of stimulus $s_{i}$, over $s_{k}$, and $c$ is a relative preference of stimulus $s_{j}$ over stimulus $s_{k}$. Matrix $A 3$ is consistent if, and only if, $b=a c$. For inconsistent matrices $b \neq a c$ and the degree of deviation from the nearest basic consistent reciprocal matrix yields an inconsistency index. This intuitive observation is the basis for the new definition of inconsistency. A matrix $A 3$ can be reduced to a vector of three coordinates $[a, b, c]$. We know that $b=a c$ holds for each consistent reciprocal matrix. Therefore, we can always produce three consistent reciprocal matrices (that is three vectors) by computing one coordinate from the combination of the remaining two coordinates. These three vectors are: $\left[\frac{b}{c}, b, c\right],[a, a c, c]$, and $\left[a, b, \frac{b}{a}\right]$. The new inconsistency index $(I x)$ can be defined as the relative distance to the nearest consistent reciprocal matrix represented by one of these three vectors for a given metric (see [7]). The inconsistency index of the basic reciprocal matrix $A 3$ is thus equal to:

$$
I x=\min \left(\frac{\left|a-\frac{b}{c}\right|}{a}, \frac{|b-a c|}{b}, \frac{\left|c-\frac{b}{a}\right|}{c}\right)
$$


Note that the inconsistency $I x$ index is not a metric. It is a matrix characteristic comparable (in nature) to entropy for a probabilistic sample space. The above definition is extended (see [2]) to reciprocal matrices of any order as

$$
I x=\min \left(\left|1-\frac{b}{a c}\right|,\left\{1-\frac{a c}{b} \mid\right) \text { for each triad }(a, b, c)\right.
$$

It is natural to use the inconsistency index of a comparisons matrix as a measure of the validity of the knowledge. To "improve" the quality of the knowledge, experts, with the help of software, compute the inconsistency of their judgments. A computer program highlights the triad with the largest inconsistency. Obviously the system does not force experts to change their judgments. Instead, the computer program flags the most critical spot in the set of judgments.

Unlike the old eigenvalue-based inconsistency ([12, 7]), the triad-based inconsistency index can be used to locate the most inconsistent triads which allows the expert to reconsider the assessments included in the most inconsistent triad. It has been shown ([6]) that the global inconsistency decreases when the local inconsistency is systematically decreased.

An important consideration is how to begin the analysis. Assigning weights to all criteria (e.g., $A=18$, $B=27, C=20, D=35$ ) seems more natural than the above process. In fact it is even a recommended practice to start with some initial values. The above values yield the ratios: $A / B=0.67, A / C=0.9$, $A / D=0.51, B / C=1.35, B / D=0.77, C / D=0.57$. Upon analysis, these may look somewhat suspicious because all of them round to 1 , the value for equal or unknown importance. This effect frequently arises in practice, and experts are tempted to change the ratios by increasing some of them and decreasing others (depending on their knowledge of the case). The changes usually cause an increase in inconsistency which, in turn, can be handled by the consistency analysis to establish more accurate and realistic weights. The pairwise comparisons method requires evaluation of all combinations of pairs of criteria, and can be time-consuming because the number of comparisons depends on $n^{2}$ (the square of the number of criteria). There is, however, an improvement in accuracy (over $300 \%$ ), as was demonstrated by a Monte Carlo experiment with bars of randomly generated lengths ([8]). The complexity problem has been addressed and partly solved by the introduction of hierarchical structures ([12]). Dividing criteria into smaller groups is a practical solution in cases where the number of criteria is large.

It is fair to say that making comparative judgements of rather intangible criteria (e.g., environmental pollu- tion or public satisfaction) results not only in imprecise knowledge, but also in inconsistency in our own judgements. In practice, inconsistent judgements are unavoidable when at least three factors are independently compared against each other.

For example, let us look closely at the ratios of the four criteria: $A, B, C$, and $D$ in Figure 2. Supposo we estimate ratios $A / B$ as $2, B / C$ as 3 , and $A / C$ as 5 . Evidently something does not "add up" because $(A / B)$. $(B / C)=2 \cdot 3=6$, which obviously is not equal to 5 (that is, $A / C)$. With an inconsistency index of 0.17 , the above described triad (with "boxed" values of 2,5 , and 3 ) is the most inconsistent in the entire matrix (reciprocal values below the main diagonal are not shown in Figure 2). A rash judgment may lead us to believe that $A / C$ should indeed be 6 , but we do not have any a priori reason to reject the estimation of $B / C$ as 2.5 or $A / B$ as $5 / 3$. After correcting $B / C$ from 3 to 2.5 (an arbitrary decision which is usually based on additional knowledgo gathering), the next most inconsistent triad is $(5,4,0.7)$ with an inconsistency index of 0.13 . An adjustmont of 0.7 to 0.8 makes this triad fully consistent $(5 \cdot 0.8$ is 4$)$, but another triad $(2.5,1.9,0.8)$ has an inconsistency of 0.05 . By changing 1.9 to 2 the entire table becomes fully consistent. The corrections for real data are done on the basis of professional experience and knowledge of the case by examining all three involved criteria.

An acceptable threshold of inconsistency is 0.33 because it means that one judgement is not more than two grades of the scale (according to Table 1) "different" from the remaining two judgments ([7]). There is no need to continue decreasing the inconsistency, as only a high value is harmful. A very small value may indicate that artificial data were entered hastily without reconsideration of former assessments.

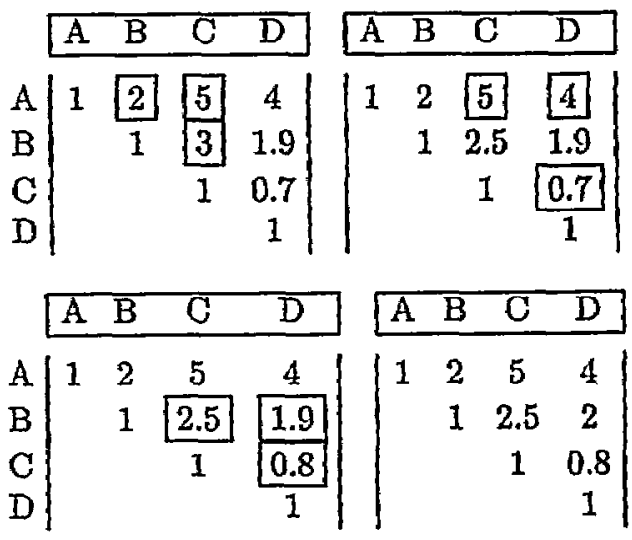

Figure 2. Process of consistency improvement,

\section{Orthogonal projections}

Algorithms for reducing the triad inconsistency in pairwise comparisons can be improved by orthogonal projections ([10]). The solution of a system of $n-2$ lin- 
ear equations produces the following orthogonal basis matrices $T_{k}=\left[t_{i j}^{k}\right]$ where

$$
t_{i j}^{k}=\left\{\begin{aligned}
-\frac{n-k}{n-k+1} & \text { for } i<k=j \\
\frac{1}{n-k+1} & \text { if } i<k<j \leq n \\
1 & \text { if } i=k<j \leq n \\
-t_{j i}^{k} & \text { if } t_{i j} \neq 0 \text { and } j<i \\
0 & \text { otherwise }
\end{aligned}\right.
$$

This is equivalent to the following simpler non-recursive definition:

$$
T_{k}=B_{k}-\frac{n-k}{n-k+1} B_{k-1}
$$

where $B_{0}$ is a matrix with all zero elements.

The matrices $B_{k}=\left[b_{i j}^{k}\right]$ (for details see Appendix A)are given by

$$
b_{i j}^{k}=\left\{\begin{aligned}
1 & \text { if } 1 \leq i \leq k<j \leq n \\
-1 & \text { if } 1 \leq j \leq k<i \leq n \\
0 & \text { otherwise }
\end{aligned}\right.
$$

The orthogonal basis for the case $n=4$ is

$$
\begin{aligned}
& T_{1}=\left|\begin{array}{rrrr}
0 & 1 & 1 & 1 \\
-1 & 0 & 0 & 0 \\
-1 & 0 & 0 & 0 \\
-1 & 0 & 0 & 0
\end{array}\right| \\
& T_{2}=\left|\begin{array}{rrrr}
0 & -\frac{2}{3} & \frac{1}{3} & \frac{1}{3} \\
\frac{2}{3} & 0 & 1 & 1 \\
-\frac{1}{3} & -1 & 0 & 0 \\
-\frac{1}{3} & -1 & 0 & 0
\end{array}\right| \\
& T_{3}=\left|\begin{array}{rrrr}
0 & 0 & -\frac{1}{2} & \frac{1}{2} \\
0 & 0 & -\frac{1}{2} & \frac{1}{2} \\
\frac{1}{2} & \frac{1}{2} & 0 & 1 \\
-\frac{1}{2} & -\frac{1}{2} & -1 & 0
\end{array}\right|
\end{aligned}
$$

Fig. 1. An example of an orthogonal basis for $n=4$

Using formula (3), for an arbitrary $n$ one can produce the $n-1$ matrices $T_{k}$, which constitute an orthogonal basis for the space $L$. Once the matrices $T_{k}$ are determined, we may compute the following values for a given matrix $A^{\prime}$ (note that the operation - is a dot product, not a regular matrix product)

$$
t_{k}=\frac{T_{k} \cdot A^{\prime}}{\left|T_{k}\right|^{2}} \quad \text { for } k=1, \ldots, n-1 \quad \text { where } A^{\prime}=\log A
$$

The triad-based consistent approximation to the ma$\operatorname{trix} A$ is then given by

$$
C=\exp C^{\prime}
$$

where $C=\sum_{k=1}^{n-1} t_{k} T_{k}$.

\section{Conclusions}

Finding an ideal vector of weights for inconsistent (or very inconsistent) matrices is a mirage. While it may be a theoretically challenging task, it does not have much practicality. It could be compared to an attempt at finding the lengths of objects using a ruler whose length changes randomly (because of, for example, temperature variations). The truth is that no "ideal" solution exists and understanding the true source of our problem, that is the inconsistency of judgments, is absolutely necessary for decreasing the inaccuracy. It is difficult to change the inconsistency without knowing the location of the most inconsistent matrix elements when only its value is known. The new definition of inconsistency locates them and experts are given necessary feedback and an opportunity to reconsider their judgments by using various approaches (e.g., Delphi method).

The Monte Carlo experiment with bars of randomly generated lengths showed a $300 \%$ improvement in accuracy in the estimation of their lengths by using pairwise comparisons. In the first part of the experiment, respondents were asked to estimate the lengths directly, whereas in the second part, respondents were asked to do the same by comparing bars in pairs. An improvement from about $15 \%$ error to $5 \%$ error was observed and verified statistically ([8]).

\section{Appendix A. The derivation of the or- thogonal basis}

Formula (3) can be obtained as follows. Given matrices $B_{1}, B_{2}, \ldots, B_{n-1}$, find $B_{1}^{\prime}, B_{2}^{\prime}, \ldots, B_{n-1}^{\prime}$ which are pairwise orthogonal and satisfy the following system of equations

$$
\begin{aligned}
& B_{1}^{\prime}=B_{1} \\
& B_{2}^{\prime}=a_{12} B_{1}+B_{2} \\
& B_{3}^{\prime}=a_{13} B_{1}+a_{23} B_{2}+B_{3} \\
& B_{4}^{\prime}=a_{14} B_{1}+a_{24} B_{2}+a_{34} B_{3} \\
& \ldots \ldots \ldots \ldots \ldots \ldots \ldots \ldots \ldots \ldots \ldots \ldots \ldots \ldots \ldots \ldots \ldots \ldots \ldots \ldots \ldots \ldots \ldots \\
& B_{n-1}^{\prime}=a_{1, n-1} B_{1}+a_{2, n-1} B_{2}+\ldots .+B_{n-1}
\end{aligned}
$$

The following properties of the above basis are helpful in the normalization

$$
\left|B_{i}\right|^{2}=2 i(n-i) \text { and } B_{i} * B_{j}=2 i(N-j) \text { if } i<j
$$

Multiply the second equation by $B_{1}$ (dot-product)

$$
0=B_{2}^{\prime} B_{1}=a_{1} 2 B_{1}^{2}+B_{2} B_{1}=a_{12} 2(n-1)+2(n-2)
$$

hence

$$
a_{12}=-\frac{n-2}{n-1}
$$


Multiply the third equation by $B_{1}$ and by $B_{2}$ (dotproduct)

$$
\begin{gathered}
0=B_{3}^{\prime} B_{1}=a_{13} B_{1}^{2}+a_{23} B_{2} B_{1}+B_{3} B_{1}= \\
a_{13} 2(n-1)+a_{23} 2(n-2)+2(n-3) \\
0=B_{3}^{\prime} B_{2}=a_{13} B_{1} B_{2}+a_{23} B_{2}^{2}+B_{3} B_{2}= \\
a_{13} 2(n-2)+a_{23} 2 * 2(n-2)+2 * 2(n-3)
\end{gathered}
$$

Solving that system of two equations, we get $a_{13}=0$ and $a_{23}=-\frac{n-3}{n-2}$

Let us consider the equation for $B_{4}$. Multiplying it by $B_{1}, B_{2}, B_{3}$ we get

$$
\begin{gathered}
0=B_{4}^{\prime} B_{1}=a_{14} B_{1}^{2}+a_{24} B_{2} B_{1}+a_{34} B_{3} B_{1}+B_{4} B_{1}= \\
a_{14} 2(n-1)+a_{24} 2(n-2)+a_{34} 2(n-3)+2(n-4) \\
0=B_{4}^{\prime} B_{2}=a_{14} B_{1} B_{2}+a_{24} B_{2}^{2}+a_{34} B_{3} B_{2}+B_{4} B_{2}= \\
a_{14} 2(n-2)+a_{24} 2 * 2(n-2)+a_{34} 2 * 2(n-3)+2 * 2(n-4) \\
0=B_{4}^{\prime} B_{3}=a_{14} B_{1} B_{3}+a_{24} B_{2} B_{3}+a_{34} B_{3}^{2}+B_{4} B_{3}= \\
a_{14} 2(n-3)+a_{24} 2 * 2(n-3)+a_{34} 2 * 3(n-3)+2 * 3(n-4)
\end{gathered}
$$

From the first two equations we calculate that $a_{14}=0$. From the second and third, after substituting 0 for $a_{14}$, we calculate $a_{24}=0$ and then $a_{34}=-\frac{n-4}{n-3}$. Similar patterns may be observed when the $k$-th equation is multiplied by vector-matrices $B_{1}, \ldots, B_{k-1}$. From these $k-1$ equations, we can see that all $a_{i k}$ are equal to 0 except $a_{k-1, k}=-\frac{n-k}{n-k+1}$ which is the coefficient in formula (3).

\section{References}

[1] Boose, J.H., A Survey of Knowledge Acquisition Techniques and Tools. Knowledge Acquisition 1, March, 1989.

[2] Duszak, Z., Koczkodaj, W.W., The Generalization of a New Definition of Consistency for Pairwise Comparisons, Information Processing Letters, 52(5), pp. 273-276, 1994.

[3] Feigenbaum, E., McCorduck, P., The Fifth Generation. Reading, MA: Addison-Wesley, 1983.

[4] Fechner, G.T., Elements of Psychophysics, Vol. 1, New York: Holt, Rinehart \& Winston, 1965, translation by H.E. Adler of Elemente der Psychophysik, Leipzig: Breitkopf und Härtel, 1860.
[5] Herman, M., Koczkodaj, W.W., Monte Carlo Study of Pairwise Comparisons, Information Processing Letters, 57(1), pp. 25-29, 1996.

[6] Holsztynski, W., Koczkodaj, W.W., Convergence Analysis of Inconsistency Algorithms for the Pairwise Comparisons Method, Information Processing Letters, 59(4), pp. 197-202, 1996.

[7] Koczkodaj, W.W., A New Definition of Consistency of Pairwise Comparisons, Mathematical and Computer Modelling, 18(7), pp. 79-84, 1993.

[8] Koczkodaj, W.W., Statistically Accurate Evidence of Impraved Error Rate by Paimuise Comparisons. Perceptual and Motor Skills, 82, 43-48, 1996.

[9] Koczkodaj, W.W., Mackasey, W.O., Mineralpotential Assessment by Consistency-driven Pairwise Comparisons, Exploration and Mining Geology, 1997 (in print)

[10] Koczkodaj, W.W., Orlowski, M., Consistent Approximation to a Pairwise Comparisons Matrix by Orthogonal Projections, (submitted)

[11] Liang, T.C., Sheng, C.L., Comments on Saaty's Consistency Ratio Measure and Proposal of a New Detecting Procedure. Journal of Information and Management Sciences, 1(2), December, pp. 55-68, 1990.

[12] Saaty, T.L., A Scaling Methods for Priorities in Hierarchical Structure, Journal of Mathematical Psychology, 15, 234-281, 1977.

[13] Shen, Y., A Probability Distribution and Convergence of the Consistency Index in the Analytic Hierarchy Process, Mathematical and Computer Modelling, 13(2), pp. 59-77, 1990.

[14] Thurstone, L.L., A Law of Comparative Judgments, Psychological Reviews, 34, 273-286, 1927.

[15] Dadkhah, K.M., Zahedi, F., A Mathematical Treatment of Inconsistency in The Analytic Hierarchy Process, Mathematical and Computer Modelling, 17(4/5), pp. 111-122, 1993.

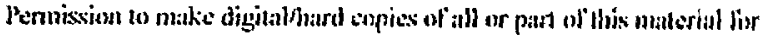

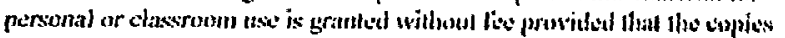

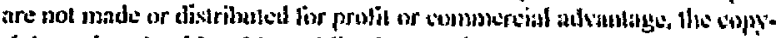

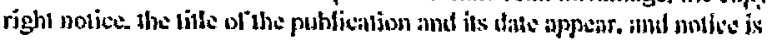

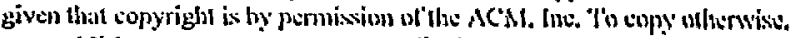

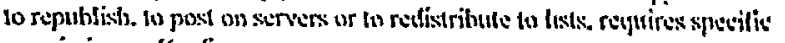
permission andror fis.

CIkM 97 Lasl egas Nevalo $1.8 \% 1$

Copyrigh 1997 AC $10-89791-970-x_{07} 11.53 .50$
} 